\title{
Applicability of fibroblast growth factor 23 for evaluation of risk of vertebral fracture and chronic kidney disease-mineral bone disease in elderly chronic kidney disease patients
}

Eiichiro Kanda ${ }^{1,2,3^{*}}$, Masayuki Yoshida ${ }^{2}$ and Sei Sasaki ${ }^{3}$

\begin{abstract}
Background: Elderly patients with chronic kidney disease (CKD) are usually at a high risk of fractures due to both osteoporosis and CKD-mineral bone disease (MBD). A new marker is needed to prevent fractures and control CKD-MBD from the early to advanced stages of CKD. In the early stage of CKD, fibroblast growth factor 23 (FGF23) level increases before parathyroid hormone (PTH) and phosphate levels increase, and steadily increases with the progression of kidney disease. It has been reported that FGF23 is related to the overall fracture risk. We investigated the usefulness of FGF23 as a marker for evaluating the risk of vertebral fracture and CKD-MBD in elderly CKD patients.
\end{abstract}

Methods: One hundred and five elderly predialysis CKD patients who had never been treated for osteoporosis and had never used calcium supplements, vitamin D supplements, or phosphate binders were enrolled in this cross-sectional study in Tokyo, Japan. We investigated the prevalence of vertebral fracture and measured serum calcium, phosphate, $1,25(\mathrm{OH})_{2}$ vitamin $\mathrm{D}\left[1,25(\mathrm{OH})_{2} \mathrm{D}\right]$, intact PTH, FGF23, alkaline phosphatase, and urinary $\mathrm{N}$-terminal telopeptide levels. Then, we examined the relationship between the level of FGF23 and those of bone-metabolism-related markers and identified markers associated with vertebral fractures in elderly CKD patients.

Results: The background features of the patients were as follows: female, 32.4\%; diabetes mellitus, 39.0\%; average age (standard deviation), 73.2 (7.7) years; and estimated glomerular filtration rate (eGFR), 45.7 (24.1) ml/min/1.73 $\mathrm{m}^{2}$. Adjusted multivariate regression analysis showed that the natural logarithm value of FGF23 level [In(FGF23)] was positively associated with body mass index $(p=0.002)$, serum phosphate level $(p=0.0001)$, and negatively with eGFR $(p=0.0006)$. Multivariate logistic regression analysis showed that vertebral fracture was independently associated with In(FGF23) (adjusted odds ratio, 4.44; 95\% confidence interval, 1.13-17.46). A

receiver-operating-characteristic curve of In(FGF23) showed that the optimal cutoff level of FGF23 indicative of vertebral fracture was $56.8 \mathrm{pg} / \mathrm{ml}$ (sensitivity, 0.82; specificity, 0.63).

Conclusions: FGF23 level was independently associated with the levels of bone-metabolism-related markers and vertebral fracture. FGF23 is a new candidate marker for detecting abnormalities of bone metabolism and vertebral fracture in elderly CKD patients.

Keywords: CKD, Fracture, FGF23, CKD-MBD, Phosphate, PTH, Geriatrics

\footnotetext{
*Correspondence: tokyo.kyosai.kanda@gmail.com

'Department of Nephrology, Tokyo Kyosai Hospital, Nakameguro 2-3-8,

Meguroku, Tokyo 153-8934, Japan

2Bioethics Research Center, Tokyo Medical and Dental University, Yushima

1-5-45, Bunkyoku, Tokyo 113-8519, Japan

Full list of author information is available at the end of the article
} 


\section{Background}

Elderly persons are at a high risk of osteoporotic fractures, because of their decreased bone mineral density (BMD). Vertebral fracture is most common [1,2]. There are several studies showing an increased fracture risk among chronic kidney disease (CKD) patients [3-6]. CKD patients tend to show a lower BMD than the general population [7]. Kidney dysfunction is associated with a more rapid decrease in BMD over time [8]. These findings indicate that elderly CKD patients are at a higher risk of fractures because of both CKD and aging than the general population.

CKD affects bone metabolism with a decreased level of the active form of vitamin $\mathrm{D}$, namely, 1,25 dihydroxy vitamin $\mathrm{D}\left[1,25(\mathrm{OH})_{2} \mathrm{D}\right]$, and an increased level of the parathyroid hormone (PTH), resulting in CKD-mineral bone disease (MBD) [9]. In CKD, fibroblast growth factor 23 (FGF23) level increases early before PTH and phosphate levels increase, and steadily increases with the progression of kidney disease [10]. The Swedish group of the Population-based Osteoporotic Fractures in Men (MrOS) study, a prospective study, showed that FGF-23 is directly associated with the overall fracture risk and vertebral fracture risk in elderly males with an average estimated glomerular filtration rate (eGFR) of $72.0 \pm 20.5 \mathrm{ml} / \mathrm{min} / 1.73 \mathrm{~m}^{2}[11]$.

Pathological abnormalities of renal osteodystrophy begin in the early stages of CKD [12]. A study on early stage CKD showed that serum calcium and phosphate levels are normal until the eGFR becomes lower than $40 \mathrm{ml} / \mathrm{min} / 1.73 \mathrm{~m}^{2}$ [13]. Serum phosphate level is not useful for monitoring CKD-MBD at an early stage of CKD, because the changes in serum phosphate level caused by therapy are usually small when the serum phosphate level is within the normal range. We need a new marker of CKD-MBD with higher sensitivity to therapy effects and higher accuracy in identifying patients at a high risk of clinical outcomes of CKD than serum phosphate level at predialysis.

Elderly individuals with CKD often have high risks of both osteoporosis and CKD-MBD. FGF23 level has a potential for identifying early-stage CKD patients that should be targeted for CKD-MBD therapy. For FGF23 level to be clinically useful, it should be demonstrated that FGF23 is a risk factor for the clinical outcomes of CKD, and the target FGF23 level for the prevention of such clinical outcomes should be determined. In this study, we investigated the prevalence of vertebral fracture that occurred within five years in predialysis CKD patients and whether FGF23 level is associated with vertebral fracture.

\section{Methods}

\section{Study design and study population}

This is a cross-sectional study of predialysis CKD patients treated at Tokyo Kyosai Hospital, Tokyo, Japan, which was approved by the Ethics Committee of Tokyo Kyosai Hospital. Patients were eligible for inclusion in the sample for this study when they were at least 60 years of age as of October 1st, 2010, diagnosed as having CKD on the basis of the criteria of the Japanese Society of Nephrology, had never been treated by dialysis or transplantation, had never been treated for osteoporosis, and had never used calcium supplements, vitamin D supplements, phosphate binders, steroids, or immunosuppressants [14]. All the female patients were postmenopausal. Patients who were treated for dementia, congestive heart failure, pulmonary disease, liver disease, or cancer were excluded. We treated CKD in accordance with the CKD practice guideline of the Japanese Society of Nephrology [14]. Body mass index (BMI) was calculated using the following formula: BMI $=\operatorname{weight}(\mathrm{kg}) / \operatorname{height}(\mathrm{m})^{2}$. eGFR was calculated individually using the formula adopted by the Japanese Society of Nephrology using serum creatinine level [14].

\section{Data}

Patient demographics including age, gender, and history of diabetes mellitus, as well as comorbid conditions, were obtained from the medical records of the patients treated at Tokyo Kyosai Hospital. Incident vertebral fractures that occurred within five years, which were diagnosed by orthopedists on the basis of X-ray, computed tomography, or magnetic resonance imaging findings, were also obtained from medical records. Intact $\mathrm{PTH}$ and $1,25(\mathrm{OH})_{2} \mathrm{D}$ levels were measured as CKD-MBDrelated markers. Urinary $\mathrm{N}$-terminal telopeptide (urinary NTX) and alkaline phosphatase (ALP) levels were measured as bone turnover markers [15-17]. Routine serum biochemistry of phosphate, calcium, serum creatinine, urinary creatinine, and intact PTH levels (1-84) was carried out by standard methods at Tokyo Kyosai Hospital. Intact PTH level was measured using an immunoassay system (ARCHITECT PTH, Abbott Japan Co., Chiba, Japan). $1,25(\mathrm{OH})_{2} \mathrm{D}$, urinary NTX, and serum intact FGF23 levels were measured by SRL Inc., Japan. 1,25 $(\mathrm{OH})_{2} \mathrm{D}$ level was analyzed using a commercially available kit, $\left(1,25(\mathrm{OH})_{2} \mathrm{D}\right.$ RIA, TFB Co., Tokyo, Japan). Serum intact FGF23 level was measured by enzymelinked immunosorbent assay (ELISA) with an intra-assay coefficient of variation (CV) of $3.8 \%$ at a low standard FGF23 level, an intra-assay CV of $2.4 \%$ at a high standard FGF23 level, and an intra-assay CV of 2.8\% (Kainos Laboratories International Co., Tokyo, Japan). Urinary NTX level was also analyzed by ELISA (OSTEOMARK, Inverness Medical Co., Chiba, Japan).

\section{Statistical analyses}

Continuous variables for normal distribution were examined by the Kolmogorov-Smirnoff test. Normally distributed 
variables are presented as mean and standard deviation (SD); otherwise, mean, SD, median and interquartile range (IQR) are presented here. For parameters not normally distributed, natural logarithm values were considered in tests that require normally distributed variables after the tests of their normality: the natural logarithm values of FGF23 level $[\ln (\mathrm{FGF} 23)]$ and PTH level $[\ln (\mathrm{iPTH})]$. Intergroup comparisons were performed using the chi-square test, t-test, and Mann-Whitney $\mathrm{U}$ test as appropriate. Pearson's correlation coefficient, $r$, was used to assess the relationship between $\ln$ (FGF23) and the levels of other bone-metabolism-related markers. Multivariate linear regression analysis was carried out to identify variables that were independently associated with $\ln$ (FGF23) by including factors that were previously selected on the basis of Pearson's correlation coefficient. Univariate logistic regression analysis was performed to identify variables that were associated with vertebral fracture. Then, multivariate logistic regression analysis was performed to identify variables that were independently associated with vertebral fracture by including factors that were previously selected on the basis of the results of the univariate logistic regression analysis. The values of odds ratio (OR) and 95\% confidence interval $(\mathrm{CI})$ are summarized. The probability of vertebral fracture occurring in each patient was predicted by calculation using a univariate logistic regression model with $\ln (\mathrm{FGF} 23)$. A receiver-operating-characteristic (ROC) curve was used to determine the optimal cutoff FGF23 level for determining the presence of vertebral fracture, at which optimal sensitivity and specificity were achieved [18]. The cutoff level was determined from the minimal value of $(1 \text {-sensitivity })^{2}+(1 \text {-specificity })^{2}$ and the Youden index. We defined $\ln$ (FGF23) to be high on the basis of the cutoff level determined from the previously obtained ROC curve. The categorized values were treated as binary numbers. We estimated the prevalence of vertebral fracture and calculated the OR of a high $\ln$ (FGF23) with respect to normal levels by univariate logistic regression and multivariate logistic regression analyses including factors that were selected on the basis of the results of the previously conducted univariate logistic regression analysis. These analyses were conducted using SAS, version 9.2 (SAS, Inc., Cary, North Carolina). Statistical significance was defined as $p<0.05$.

\section{Results}

One hundred and five elderly CKD patients were included in the sample for analysis. Patient demographics including biochemical data are shown in Table 1. The causes of CKD were as follows: diabetic nephropathy,

Table 1 Baseline characteristics of patients with vertebral fracture in comparison with those without the fracture

\begin{tabular}{|c|c|c|c|c|}
\hline & All & With-fracture group & Without-fracture group & $p$ value \\
\hline$N(\%)$ & 105 & $11(10.5)$ & $94(89.5)$ & \\
\hline Age (years) & $73.2(7.7)$ & $79.0(6.6)$ & $72.5(7.5)$ & 0.008 \\
\hline Female (\%) & $34(32.4)$ & $5(45.5)$ & $29(30.9)$ & 0.33 \\
\hline Diabetes mellitus (\%) & $41(39.0)$ & $4(36.4)$ & $37(39.4)$ & 0.84 \\
\hline Height (cm) & $159.5(6.9)$ & $161.1(3.7)$ & $159.2(7.2)$ & 0.41 \\
\hline Weight (kg) & $61.3(8.6)$ & $63.2(2.5)$ & $61.0(9.2)$ & 0.44 \\
\hline $\mathrm{BMI}\left(\mathrm{kg} / \mathrm{m}^{2}\right)$ & $24.0(2.5)$ & $24.4(1.5)$ & $24.0(2.6)$ & 0.60 \\
\hline eGFR $\left(\mathrm{ml} / \mathrm{min} / 1.73 \mathrm{~m}^{2}\right)$ & $45.7(24.1)$ & $32.9(18.8)$ & $47.2(24.3)$ & 0.063 \\
\hline Calcium level (mmol/l) & $2.4(0.1)$ & $2.3(0.2)$ & $2.4(0.1)$ & 0.85 \\
\hline Phosphate level (mmol/l) & $1.1(0.2)$ & $1.1(0.2)$ & $1.1(0.2)$ & 0.81 \\
\hline Intact PTH level (ng/l) & $\begin{array}{c}143.6(139.4) \\
96.2(\text { IQR, 65.8-154.4) }\end{array}$ & $\begin{array}{c}142.5(79.3) \\
76(\text { IQR, 45-214) }\end{array}$ & $\begin{array}{c}143.6(145.1) \\
91.3(\text { (IQR, 65.1-154.4) }\end{array}$ & 0.29 \\
\hline Ln(iPTH) & $4.7(0.7)$ & $4.8(0.5)$ & $4.7(0.7)$ & 0.60 \\
\hline $1,25(\mathrm{OH})_{2} \mathrm{D}$ level $(\mathrm{pmol} / \mathrm{l})$ & $122.6(54.6)$ & $88.3(49.0)$ & $126.8(54.0)$ & 0.026 \\
\hline FGF23 level (pg/ml) & $\begin{array}{c}78.0(101.7) \\
49.0(\mathrm{IQR}, 34.0-71.0)\end{array}$ & $\begin{array}{c}136.2(123.2) \\
120.7(\text { IQR, 82.2-179.1) }\end{array}$ & $\begin{array}{c}71.2(97.4) \\
47(\mathrm{IQR}, 34-66)\end{array}$ & 0.012 \\
\hline $\operatorname{Ln}(F G F 23)$ & $4.0(0.7)$ & $4.6(0.8)$ & $3.9(0.7)$ & 0.005 \\
\hline ALP level (U/I) & $227.0(83.1)$ & $252.1(103.5)$ & $224.1(80.5)$ & 0.29 \\
\hline Urinary NTX level (nmol.BCE/mmol.Cr) & $35.8(24.3)$ & $36.9(27.3)$ & $35.6(24.1)$ & 0.88 \\
\hline
\end{tabular}

Values are expressed as mean (SD). The levels of intact PTH and FGF23 are presented with mean (SD), and median (IQR). The levels are compared between the groups by the chi-square test, t-test, or Mann-Whitney $\mathrm{U}$ test as appropriate.

Abbreviations: with-fracture group, group with vertebral fracture; without-fracture group, group without vertebral fracture; BMI body mass index; eGFR estimated glomerular filtration rate; intact PTH intact parathyroid hormone; In(iPTH) natural logarithm value of intact parathyroid hormone level; $1,25(\mathrm{OH})_{2} \mathrm{D} 1,25(\mathrm{OH})_{2}$ vitamin D; FGF23 fibroblast growth factor 23; In(FGF23) natural logarithm value of fibroblast growth factor 23 level; $A L P$ alkaline phosphatase; urinary NTX urinary $\mathrm{N}$-terminal telopeptide; $S D$ standard deviation; $I Q R$ interquartile range. 
39.0\%; nephrosclerosis, $17.3 \%$; chronic glomerulonephritis, $35.6 \%$; and others, $8.1 \%$. Eleven patients were diagnosed as having vertebral fracture (10.5\%). Intact PTH and FGF23 levels were log-normally distributed: mean (SD) intact PTH level, 143.6 (139.4) ng/l with a median of 96.2 (IQR, 65.8-154.4); FGF23 level, 78.0 (101.7) pg/ml with a median of 49.0 (IQR, 34.0-71.0).

Groups with and without fractures were balanced for gender, diabetes, BMI, eGFR, serum calcium level, serum phosphate level, intact PTH level, ALP level, and urinary NTX level. The average age of the with-fracture group was higher than that of the without-fracture group. The with-fracture group showed a lower $1,25(\mathrm{OH})_{2} \mathrm{D}$ level and a higher FGF23 level than the without-fracture group.

\section{FGF23 and markers of bone metabolism}

There was a correlation between $\ln$ (FGF23) and the levels of bone-metabolism-related markers (Table 2). Ln (FGF23) was positively associated with age, BMI, serum phosphate level, and $\ln (\mathrm{iPTH})$, and urinary NTX level, and negatively with eGFR, serum calcium level, and 1,25 $(\mathrm{OH})_{2} \mathrm{D}$ level. Multivariate regression analysis of the variables age, BMI, eGFR, calcium level, phosphate level, $\ln (\mathrm{iPTH}), \quad 1,25(\mathrm{OH})_{2} \mathrm{D}$ level, and urinary NTX level showed that $\ln$ (FGF23) was independently associated with BMI, eGFR, and serum phosphate level.

\section{FGF23 and vertebral fracture}

The associations between vertebral fracture and the levels of bone-metabolism-related markers are shown in Table 3. Univariate logistic regression analysis showed

Table 2 Ln(FGF23) correlated with levels of bonemetabolism-related markers

\begin{tabular}{|c|c|c|c|c|}
\hline & \multicolumn{2}{|c|}{$\begin{array}{c}\text { Pearson's correlation } \\
\text { coefficient }\end{array}$} & \multicolumn{2}{|c|}{$\begin{array}{c}\text { Multiple regression } \\
\text { analysis }\end{array}$} \\
\hline & $r$ & $p$ & $\beta$ & $p$ \\
\hline Age & 0.22 & 0.026 & -0.006 & 0.31 \\
\hline $\mathrm{BMI}$ & 0.24 & 0.019 & 0.056 & 0.002 \\
\hline eGFR & -0.73 & 0.0001 & -0.012 & 0.0006 \\
\hline Calcium level & -0.33 & 0.0006 & -0.53 & 0.23 \\
\hline Phosphate level & 0.48 & 0.0001 & 0.90 & 0.0001 \\
\hline Ln(iPTH) & 0.68 & 0.0001 & 0.16 & 0.12 \\
\hline $1,25(\mathrm{OH})_{2} \mathrm{D}$ level & -0.63 & 0.0001 & -0.0022 & 0.08 \\
\hline ALP level & 0.19 & 0.06 & & \\
\hline Urinary NTX level & 0.26 & 0.009 & 0.0014 & 0.47 \\
\hline
\end{tabular}

Values are expressed as $r$ (Pearson's correlation coefficient), $\beta$ (estimated parameter), and $p$ values.

Multiple regression analysis of age, eGFR, calcium level, phosphate level, In (iPTH), and 1,25(OH)2D level as variables.

Abbreviations: In (FGF23) natural logarithm value of fibroblast growth factor 23 level; BMI body mass index; eGFR estimated glomerular filtration rate; $\ln (\mathrm{IPTH})$ natural logarithm value of intact parathyroid hormone level; $1,25(\mathrm{OH})_{2} \mathrm{D} 1,25$ $(\mathrm{OH})_{2}$ vitamin D; FGF23 fibroblast growth factor 23; ALP alkaline phosphatase; urinary NTX urinary N-terminal telopeptide.
Table 3 Vertebral fracture correlated with background features, eGFR, and levels of bone-metabolism-related markers

\begin{tabular}{lll}
\hline & $\begin{array}{l}\text { Univariate } \\
\text { logistic } \\
\text { regression } \\
\text { OR }(\mathbf{9 5 \%} \mathbf{C I})\end{array}$ & $\begin{array}{l}\text { Multivariate } \\
\text { logistic } \\
\text { regression } \\
\text { OR (95\% CI) }\end{array}$ \\
\hline Age (per increase in 1 year) & $1.13(1.03-1.24)$ & $1.13(1.02-1.26)$ \\
Female (Male is reference) & $1.87(0.53-6.62)$ & \\
$\begin{array}{l}\text { Diabetes mellitus } \\
\text { (nondiabetes is reference) }\end{array}$ & $0.88(0.24-3.22)$ & \\
BMI (per increase in 1) & $1.07(0.83-1.37)$ & \\
eGFR (per increase in & $0.97(0.95-1.00)$ & $1.03(0.98-1.08)$
\end{tabular}

$1 \mathrm{ml} / \mathrm{min} / 1.73 \mathrm{~m}^{2}$ )

Calcium level

(per increase in $1 \mathrm{mmol} / \mathrm{l}$ )

Phosphate level

(per increase in $1 \mathrm{mmol} / \mathrm{l}$ )

$0.63(0.005-72.0)$

$1.38(0.10-18.86)$

$\mathrm{Ln}(\mathrm{iPTH})$ (per increase in 1)

$1.38(0.59-3.23)$

$1,25(\mathrm{OH})_{2} \mathrm{D}$ level

$0.98(0.97-1.00)$

(per increase in $1 \mathrm{pmol} / \mathrm{l}$ )

$2.70(1.27-5.74)$

$4.44(1.13-17.46)$

Ln(FGF23) (per increase in 1)

ALP level (per increase in $1 \mathrm{U} / \mathrm{I}$ )

$1.00(0.99-1.01)$

$1.00(0.98-1.03)$

Urinary NTX level (per increase in $1 \mathrm{nmol} . \mathrm{BCE} / \mathrm{mmol} . \mathrm{Cr}$ )

Multivariate logistic regression OR was determined from a logistic regression model that includes age, eGFR, and $\ln (\mathrm{FGF} 23)$ as variables.

Abbreviations: $O R$ odds ratio; $C l$ confidence interval; $B M I$ body mass index; eGFR estimated glomerular filtration rate; $\ln (\mathrm{iPTH})$ natural logarithm value of intact parathyroid hormone level; $1,25(\mathrm{OH})_{2} \mathrm{D} 1,25(\mathrm{OH})_{2}$ vitamin $\mathrm{D} ; \operatorname{In}(\mathrm{FGF} 23)$ natural logarithm value of fibroblast growth factor 23 level; $A L P$ alkaline phosphatase; urinary NTX urinary N-terminal telopeptide.

that vertebral fracture was associated with age, eGFR, and $\ln$ (FGF23). The estimated probability obtained using a univariate logistic model with $\ln (\mathrm{FGF} 23)$ was used to assess the effect of FGF23 level on vertebral fracture (Figure 1). The estimated probability of vertebral fracture tended to increase with FGF23 level. A multivariate logistic regression model including age, eGFR, and $\ln$ (FGF23) showed that $\ln$ (FGF23) was independently associated with vertebral fracture.

The ROC curve shows the accuracy of $\ln$ (FGF23) in identifying patients at different prevalence rates of vertebral fracture examined in all the participants (Figure 2). At the cutoff level, $\ln$ (FGF23) was 4.04 (FGF23 level $=56.8 \mathrm{pg} / \mathrm{ml}$ ): sensitivity, 0.82; specificity, 0.63; area under the curve (AUC), 0.787. Univariate and multivariate logistic regression analyses showed associations between the likelihood of vertebral fracture and high $\ln$ (FGF23): OR, 6.62 (CI, 1.63-26.84); OR adjusted for age and eGFR, 7.91 (CI, 1.09-57.56).

\section{Discussion}

CKD and osteoporosis are common diseases associated with aging, and the number of people with these 


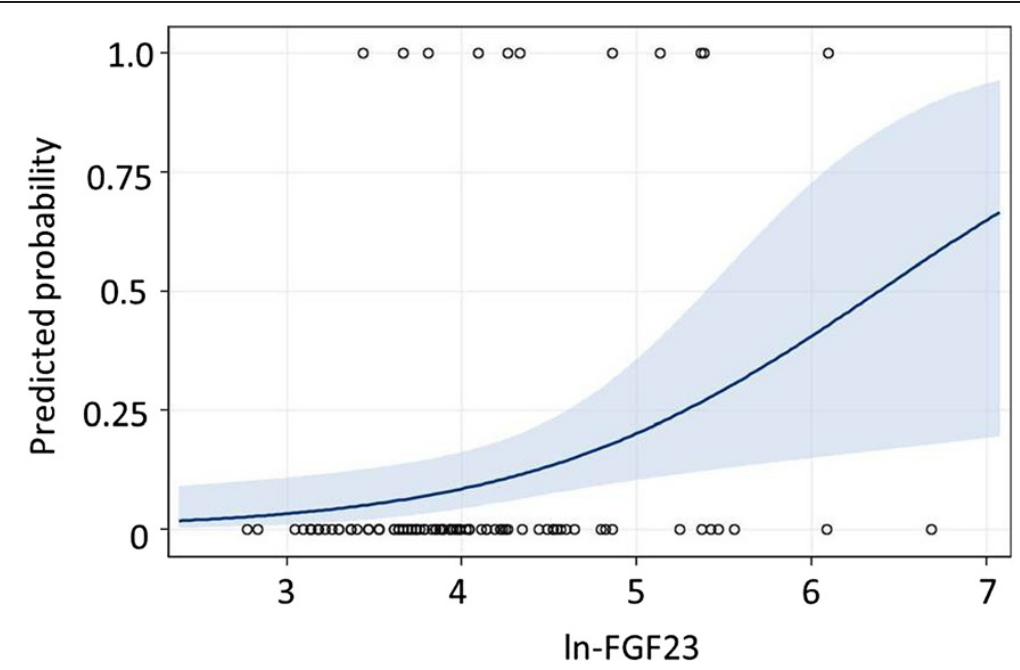

Figure 1 Predicted probability of detecting vertebral fracture increases with FGF23 level. A dot shows whether a patient has vertebral fracture (predicted probability $=1$ ) or not (predicted probability $=0$ ). A line shows the predicted probability of detecting vertebral fracture in a patient. The blue area is the $95 \%$ confidence interval. Abbreviations: estimated probability, estimated probability of occurrence of vertebral fracture; In(FGF23), natural logarithm value of fibroblast growth factor 23 level.

diseases will increase as will the risk of fractures. However, there are still many unresolved issues about these diseases: the details about bone metabolism in patients with these diseases and the identification of patients with a high risk of fractures. Our findings are consistent with previous reports that the FGF23 level is high in CKD patients with decreased eGFR, increased PTH level, and decreased $1,25(\mathrm{OH})_{2} \mathrm{D}$ level [19]. We found that FGF23 level was independently associated with vertebral fracture that occurred within five years in elderly

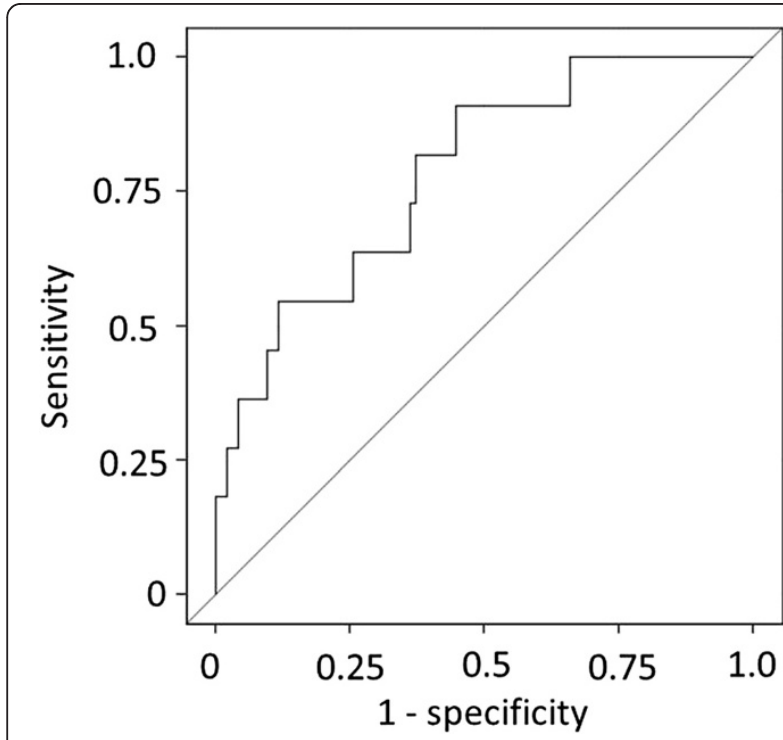

Figure 2 Receiver operating characteristic curve for In(FGF23) at optimal cutoff level for predicting vertebral fracture.

Abbreviations: In(FGF23), natural logarithm value of fibroblast growth factor 23 level.
CKD patients. The MrOS study showed prospectively that FGF23 level is related to fracture risk in elderly CKD male patients [11]. In this study, no statistically significant difference in the risk of vertebral fracture was found between genders. These findings can be extended to the association between FGF23 level and vertebral fracture in both sexes with advanced CKD. Moreover, we were able to determine the cutoff FGF23 level for identifying the patients with a high risk of vertebral fracture.

Mutations of FGF23-related genes are associated with several human skeletal disorders. It has been shown that low FGF23 activity due to mutations in GALNT3, FGF23, and Klotho leads to familial hyperphosphatemic tumoral calcinosis, which is characterized by enhanced renal tubular phosphate reabsorption and high serum $1,25(\mathrm{OH})_{2} \mathrm{D}$ level in hyperphosphatemia [20-22]. Some types of rickets and osteomalacia such as X-linked hypophosphatemic rickets/osteomalacia, hypophosphatemic rickets/osteomalacia associated with McCune-Albright syndrome/fibrous dysplasia, and tumor-induced rickets/ osteomalacia show hypophosphatemia and an increased FGF23 level [23-27]. Overexpressed human FGF23 during osteoblast development in fetal rat calvaria cells suppresses not only osteoblast differentiation but also matrix mineralization independently of its systemic effects on phosphate homeostasis in vitro [28]. A rickets mouse model shows defective osteocyte maturation, an increased FGF23 expression level, and pathological changes in bone mineralization [29]. These clinical and experimental findings suggest that an increased FGF23 level in CKD patients may affect the regulation of bone formation and reabsorption. However, these diseases and 
manifestations in animal models were also observed in humans or animals with normal kidney function. The role of FGF23 in skeletal mineralization remains unclarified in CKD patients. The high level of FGF23 in CKD is considered to further increase as a compensatory response to maintain phosphate balance because of suppressed renal phosphate excretion or phosphate overload $[30,31]$. It has been reported that high FGF23 levels are associated with thin osteoids and a short osteoid maturation time in children with renal failure on peritoneal dialysis [32]. Although these findings are not fully applicable to elderly CKD patients, this report suggests that FGF23 indicates the skeletal mineralization status in CKD patients. FGF23 could be a marker of CKD-MBD.

In this study, ROC showed that the cutoff FGF23 level was $56.8 \mathrm{pg} / \mathrm{ml}$. The adjusted logistic regression analysis showed that FGF23 level was an independent factor associated with the prevalence of vertebral fracture that occurred within five years. As far as we researched, there is only one report about the cutoff level for detecting the risk of fractures. The MrOS study showed prospectively that FGF23 levels higher than $55.7 \mathrm{pg} / \mathrm{ml}$ are associated with an increased risk of hip and nonvertebral fractures and that FGF23 levels higher than $57.4 \mathrm{pg} / \mathrm{ml}$ are associated with an increased risk of vertebral fracture [11]. They used the same ELISA kit as in our study, and these cutoff levels are similar. These two studies differed in the population with regard to age, gender, GFR, and location. Age is an independent risk factor for vertebral fracture $[1,2]$. GFR is associated with BMD $[7,8]$ and a major predictor of FGF23 level variance in CKD patients [33]. Although these findings indicate the importance of FGF23 level for detecting CKD patients at a high risk of fractures, they do not fully support the validity of these cutoff levels among genreal CKD patients because of the confounding effect of eGFR. The difference in FGF23 level between CKD populations has not been established yet. To determine the cutoff FGF23 level for clinical applications, larger population-based prospective studies need to be carried out.

According to bone biopsy studies, pathological abnormalities in renal osteodystrophy begin in the early stages of CKD [12]. However, the optimal management of CKD-MBD including the monitoring of the levels of markers and therapies initiated at the early stages of CKD has not been established yet. Our study showed that $\ln$ (FGF23) was independently positively associated with CKD-MBD-related markers and negatively with eGFR. It has been reported that increased FGF23 levels are associated with an increased risk of progressive CKD independently of normal serum phosphate level [34]. Moreover, an increased FGF23 level is more predictive of CKD progression than serum phosphate level [34]. The changes in serum phosphate level within the normal range tend to not sensitively reflect CKD-MBD in early stages of CKD. On the other hand, FGF23 and PTH levels are often elevated in CKD, but it remains undetermined which increases first and whether the pattern is uniform across all CKD patients. According to the Chronic Renal Insufficiency Cohort Study, FGF23 level increases before PTH and phosphate levels increase in CKD patients [10]. Therefore, FGF23 level can be one of the candidate new markers, because it reflects the progression of CKD-MBD, can be easily followed up longitudinally from the early stages of CKD, and can predict outcomes. The AUC of our study was 0.787 , which indicates a moderate accuracy [18]. Although this accuracy may not be very high for FGF23 level to be used as a gold standard for identifying patients with a risk of vertebral fracture, FGF23 level together with the levels of other bone-metabolism-related markers and dualenergy X-ray absorptiometry will be a more useful tool than FGF23 level alone for evaluating CKD-MBD and the risk for fractures and for deciding medications for CKD patients to prevent fractures and progression of CKD-MBD.

This study has several limitations. First, as with any cross-sectional study, we were unable to examine the longitudinal changes in laboratory findings over time. Nonetheless, this study showed that FGF23 level is independently associated with vertebral fractures that already occurred. Second, in this study, we examined 105 patients. The statistical power of this study may not be sufficient for detecting the relationship between fractures and levels of markers. Third, geographical and selection biases may have been included in this study. Fourth, BMD, bone turnover, and mineralization were not measured by an accurate method, such as bone biopsy, in this population. We were unable to evaluate the relationship between FGF23 level and BMD. Fifth, we did not evaluate the medicines used and their effects on the prognosis of kidney function. Sixth, 25-hydroxy vitamin D level was not measured in this study. We were unable to evaluate the CKD patients' status of vitamin D metabolism. Seventh, only postmenopausal females were enrolled in this study. Thus, we were unable to determine the role of FGF23 in menopausal or premenopausal females.

\section{Conclusions}

For elderly CKD patients, age-related bone diseases and CKD-MBD are significant issues, because such diseases strongly affect their quality of life and mortality. However, there has been no strong evidence that can fully establish indicators that can help guide therapies for bone diseases. We found that the level of FGF23 was associated with those of CKD-MBD-related markers and vertebral fracture in elderly CKD patients. FGF23 level 
may be a useful marker for detecting and preventing abnormalities of bone metabolism and vertebral fractures from the early to advanced stages of CKD.

\section{Abbreviations}

CKD: Chronic kidney disease; MBD: Mineral bone disease; FGF23: Fibroblast growth factor 23; PTH: Parathyroid hormone; 1,25(OH) 2 D: 1,25(OH) 2 vitamin D; eGFR: Estimated glomerular filtration rate; In(FGF23): Natural logarithm value of FGF23 level; BMD: Bone mineral density; MrOS study: The Swedish group of the Population-based Osteoporotic Fractures in Men Study; BMI: Body mass index; Urinary NTX: Urinary N-terminal telopeptide; ALP: Alkaline phosphatase; ELISA: Enzyme-linked immunosorbent assay; CV: Coefficient of variation; SD: Standard deviation; IQR: $25^{\text {th }}$ to $75^{\text {th }}$ intrequartile range; In(iPTH): Natural logarithm value of intact PTH level; OR: Odds ratio; Cl: Confidence interval; ROC: Receiver-operating-characteristic; AUC: Area under the curve.

\section{Competing interests}

No financial or other interests to be declared.

\section{Authors' contributions}

EK, MY, and SS conceptualized the study and its objective. EK and MY designed the study, extracted the data from the TK database, analyzed the data statistically, and contributed in the interpretation of the results. EK wrote the manuscript. MY and SS revised the manuscript critically and contributed substantially to the content of the article. All the authors read and approved the final manuscript.

\section{Acknowledgements}

We thank Dr. M. Ai, Bioethics Research Center, Tokyo Medical and Dental University, for his help in statistical analyses. This study was not supported by any grant.

\section{Author details}

'Department of Nephrology, Tokyo Kyosai Hospital, Nakameguro 2-3-8, Meguroku, Tokyo 153-8934, Japan. Bioethics Research Center, Tokyo Medical and Dental University, Yushima 1-5-45, Bunkyoku, Tokyo 113-8519, Japan. ${ }^{3}$ Department of Nephrology, Tokyo Medical and Dental University, Yushima 1-5-45, Bunkyoku, Tokyo 113-8519, Japan.

Received: 24 March 2012 Accepted: 23 September 2012 Published: 26 September 2012

\section{References}

1. Pøodenphant J, Nielsen VA, Riis BJ, Gotfredsen A, Christiansen C: Bone mass, bone structure and vertebral fractures in osteoporotic patients. Bone 1987, 8(3):127-130.

2. Riggs BL, Melton LJ: Involutional osteoporosis. N Engl J Med 1986, 314(26):1676-1686.

3. Ensrud KE, Lui LY, Taylor BC, Ishani A, Shlipak MG, Stone KL, Cauley JA, Jamal SA, Antoniucci DM, Cummings SR, et al: Renal function and risk of hip and vertebral fractures in older women. Arch Intern Med 2007, 167(2):133-139.

4. Fried LF, Biggs ML, Shlipak MG, Seliger S, Kestenbaum B, Stehman-Breen C, Sarnak M, Siscovick D, Harris T, Cauley J, et al: Association of kidney function with incident hip fracture in older adults. J Am Soc Nephrol 2007, 18(1):282-286

5. Nickolas TL, McMahon DJ, Shane E: Relationship between moderate to severe kidney disease and hip fracture in the United States. J Am Soc Nephrol 2006, 17(11):3223-3232.

6. Nickolas TL, Stein E, Cohen A, Thomas V, Staron RB, McMahon DJ, Leonard $M B$, Shane $E$ : Bone mass and microarchitecture in CKD patients with fracture. J Am Soc Nephrol 2010, 21(8):1371-1380.

7. Klawansky S, Komaroff E, Cavanaugh PF, Mitchell DY, Gordon MJ, Connelly JE, Ross SD: Relationship between age, renal function and bone mineral density in the US population. Osteoporos Int 2003, 14(7):570-576.

8. Fried LF, Shlipak MG, Stehman-Breen C, Mittalhenkle A, Seliger S, Sarnak M, Robbins J, Siscovick D, Harris TB, Newman AB, et al: Kidney function predicts the rate of bone loss in older individuals: the Cardiovascular Health Study. J Gerontol A Biol Sci Med Sci 2006, 61(7):743-748.
9. Foundation NK: K/DOQI clinical practice guidelines for bone metabolism and disease in chronic kidney disease. Am J Kidney Dis 2003, 42(4 Suppl 3):S1-S201

10. Isakova T, Wahl P, Vargas GS, Gutiérrez OM, Scialla J, Xie H, Appleby D, Nessel L, Bellovich K, Chen J, et al: Fibroblast growth factor 23 is elevated before parathyroid hormone and phosphate in chronic kidney disease. Kidney Int 2011, 79(12):1370-1378.

11. Mirza MA, Karlsson MK, Mellström D, Orwoll E, Ohlsson C, Ljunggren O, Larsson TE: Serum fibroblast growth factor-23 (FGF-23) and fracture risk in elderly men. J Bone Miner Res 2011, 26(4):857-864.

12. Hamdy NA, Kanis JA, Beneton MN, Brown CB, Juttmann JR, Jordans JG Josse S, Meyrier A, Lins RL, Fairey IT: Effect of alfacalcidol on natural course of renal bone disease in mild to moderate renal failure. BMJ 1995, 310(6976):358-363.

13. Levin A, Bakris GL, Molitch M, Smulders M, Tian J, Williams LA, Andress DL: Prevalence of abnormal serum vitamin D, PTH, calcium, and phosphorus in patients with chronic kidney disease: results of the study to evaluate early kidney disease. Kidney Int 2007, 71(1):31-38.

14. Nephrology JS: Evidence-based practice guideline for the treatment of CKD. Clin Exp Nephrol 2009, 13(6):537-566

15. Schneider DL, Barrett-Connor EL: Urinary N-telopeptide levels discriminate normal, osteopenic, and osteoporotic bone mineral density. Arch Intern Med 1997, 157(11):1241-1245.

16. Hanson DA, Weis MA, Bollen AM, Maslan SL, Singer FR, Eyre DR: A specific immunoassay for monitoring human bone resorption: quantitation of type I collagen cross-linked N-telopeptides in urine. J Bone Miner Res 1992, 7(11):1251-1258.

17. Tolouian R, Hernandez GT, Chiang WY, Gupta A: A new approach for evaluating bone turnover in chronic kidney disease. Eur J Intern Med 2010, 21(3):230-232.

18. Akobeng AK: Understanding diagnostic tests 3: Receiver operating characteristic curves. Acta Paediatr 2007, 96(5):644-647.

19. Gutiérrez OM: Fibroblast growth factor 23 and disordered vitamin D metabolism in chronic kidney disease: updating the "trade-off" hypothesis. Clin J Am Soc Nephrol 2010, 5(9):1710-1716.

20. Larsson T, Davis SI, Garringer HJ, Mooney SD, Draman MS, Cullen MJ, White KE: Fibroblast growth factor-23 mutants causing familial tumoral calcinosis are differentially processed. Endocrinology 2005 146(9):3883-3891.

21. Topaz $O$, Shurman $D L$, Bergman $R$, Indelman M, Ratajczak $P$, Mizrachi $M$, Khamaysi Z, Behar D, Petronius D, Friedman V, et al: Mutations in GALNT3, encoding a protein involved in O-linked glycosylation, cause familial tumoral calcinosis. Nat Genet 2004, 36(6):579-581.

22. Ichikawa S, Imel EA, Kreiter ML, Yu X, Mackenzie DS, Sorenson AH, Goetz R, Mohammadi M, White KE, Econs MJ: A homozygous missense mutation in human KLOTHO causes severe tumoral calcinosis. J Clin Invest 2007 117(9):2684-2691.

23. Consortium A: Autosomal dominant hypophosphataemic rickets is associated with mutations in FGF23. Nat Genet 2000, 26(3):345-348.

24. Lorenz-Depiereux B, Bastepe $M$, Benet-Pagès $A$, Amyere $M$, Wagenstaller J, Müller-Barth U, Badenhoop K, Kaiser SM, Rittmaster RS, Shlossberg AH, et al: DMP1 mutations in autosomal recessive hypophosphatemia implicate a bone matrix protein in the regulation of phosphate homeostasis. Nat Genet 2006, 38(11):1248-1250.

25. Francis F, Hennig S, Korn B, Reinhardt R, de Jong P, Poustka A, Lehrach $H_{\text {, }}$ Rowe PSN, Goulding JN, Summerfield T, Mountford R, Read AP, Popowska E, Pronicka E, Davies KE, O'Riordan JLH, Econs MJ, Nesbitt T, Drezner MK, Oudet C, Pannetier S, Hanauer A, Strom TM, Meindl A, Lorenz B, Cagnoli B, Mohnike KL, Murken J, Meitinger T: A gene (PEX) with homologies to endopeptidases is mutated in patients with $\mathrm{X}$-linked hypophosphatemic rickets. The HYP Consortium. Nat Genet 1995, 11(2):130-136.

26. Riminucci M, Collins MT, Fedarko NS, Cherman N, Corsi A, White KE, Waguespack S, Gupta A, Hannon T, Econs MJ, et al: FGF-23 in fibrous dysplasia of bone and its relationship to renal phosphate wasting. J Clin Invest 2003, 112(5):683-692.

27. Shimada T, Mizutani S, Muto T, Yoneya T, Hino R, Takeda S, Takeuchi Y, Fujita T, Fukumoto S, Yamashita T: Cloning and characterization of FGF23 as a causative factor of tumor-induced osteomalacia. Proc Natl Acad Sci USA 2001, 98(11):6500-6505.

28. Wang H, Yoshiko Y, Yamamoto R, Minamizaki T, Kozai K, Tanne K, Aubin JE, Maeda N: Overexpression of fibroblast growth factor 23 suppresses 
osteoblast differentiation and matrix mineralization in vitro. $J$ Bone Miner Res 2008, 23(6):939-948.

29. Feng JQ, Ward LM, Liu S, Lu Y, Xie Y, Yuan B, Yu X, Rauch F, Davis SI, Zhang S, et al: Loss of DMP1 causes rickets and osteomalacia and identifies a role for osteocytes in mineral metabolism. Nat Genet 2006, 38(11):1310-1315.

30. Shigematsu T, Kazama JJ, Yamashita T, Fukumoto S, Hosoya T, Gejyo F, Fukagawa M: Possible involvement of circulating fibroblast growth factor 23 in the development of secondary hyperparathyroidism associated with renal insufficiency. Am J Kidney Dis 2004, 44(2):250-256.

31. Di lorio B, Di Micco L, Torraca S, Sirico ML, Russo L, Pota A, Mirenghi F, Russo D: Acute effects of very-low-protein diet on FGF23 levels: a randomized study. Clin J Am Soc Nephrol 2012, 7(4):581-587.

32. Wesseling-Perry K, Pereira RC, Wang H, Elashoff RM, Sahney S, Gales B, Jüppner H, Salusky IB: Relationship between plasma fibroblast growth factor- 23 concentration and bone mineralization in children with renal failure on peritoneal dialysis. J Clin Endocrinol Metab 2009, 94(2):511-517.

33. Filler G, Liu D, Huang SH, Casier S, Chau LA, Madrenas J: Impaired GFR is the most important determinant for FGF-23 increase in chronic kidney disease. Clin Biochem 2011, 44(5-6):435-437.

34. Fliser D, Kollerits B, Neyer U, Ankerst DP, Lhotta K, Lingenhel A, Ritz E, Kronenberg F, Kuen E, König P, et al: Fibroblast growth factor 23 (FGF23) predicts progression of chronic kidney disease: the Mild to Moderate Kidney Disease (MMKD) Study. J Am Soc Nephrol 2007, 18(9):2600-2608.

doi:10.1186/1471-2369-13-122

Cite this article as: Kanda et al:: Applicability of fibroblast growth factor 23 for evaluation of risk of vertebral fracture and chronic kidney

disease-mineral bone disease in elderly chronic kidney disease patients. BMC Nephrology 2012 13:122.

\section{Submit your next manuscript to BioMed Central and take full advantage of:}

- Convenient online submission

- Thorough peer review

- No space constraints or color figure charges

- Immediate publication on acceptance

- Inclusion in PubMed, CAS, Scopus and Google Scholar

- Research which is freely available for redistribution 